\title{
Users/consumers differences regarding ergonomics and design theory and practice
}

\author{
Pierre-Henri Dejean and Peter Wagstaff \\ Chairman of IEA Ergonomics in Design Technical Committee, Université de Technologie de Compiègne \\ pierre-henri.dejean@utc.fr \\ Consultant R\&D, CEREMH, Centre of Resources and Innovation Mobility and Handicap.Vélizy, \\ peterwag@gmail.com
}

\begin{abstract}
This paper presents the concept of direct and indirect users, a key issue to cooperation between ergonomists, designers and managers involved in a sustainable approach to design. What issues for Ergonomics and Design are launched by this concept? User/consumer differences should be approached taking into account Ergonomics and Design theory and practice. What dialogue and tools could help the ergonomist/designer/manager to respond to all the requirements of the future clients of the product?
\end{abstract}

Keywords: Ergonomics/design/management cooperation, User approach, Design process certification.

\section{Why ask this question?}

The same words do not have the same meaning in different professional fields, since the user is the client targeted by marketing; the final user by the designer and the project manager has to consider both direct and indirect users. What kind of users do the Designer and the Ergonomist have to consider during their work? This is the main issue addressed in this paper. Considering the product life cycle and considerations of Quality and Sustainable development, ergonomists and designers have to take care of the well being of different kinds of persons. This duty implies the addition of different kinds of constraints, interests, culture, values and other factors. During several EQUID meetings we have talked about this issue and understand that is it not evident to everyone. In order to establish a framework for this approach we will talk about direct and indirect users (Caliste, Dejean 2012) with the intention of finding an efficient manner to summarize the large range of clients who have to be considered in the design process. In practice this means how we can introduce the needs of different categories into the design process: experts, focus groups, user models, and web persona such as designer/user dialogues.
In the first section we will introduce the concept and develop some links between the points of view of Quality, Ethics, project Management and Marketing, and in the second we will develop some practical issues, such as how to find direct and indirect users, how to consider them in the design process, and what methods and tools could help us to do that.

\section{The concept}

The key to a democratic society is that everyone should respect everyone else. Any action or behavior of a person should not disturb the well being of others. Furthermore, the concept of the respect of ecology introduces an additional stakeholder into this complex system in the form of the environment, which should also be respected by everyone. Identifying which are the stakeholders that the ergonomist and designer have to consider and how to find them is the first task. In order to explain this concept and to begin to propose a framework to applied to it we will discuss the features that determine who are direct and indirect users in addition to including environmental considerations which are another way of affecting indirect users. 


\section{Direct and indirect users}

The first important question in analysing a situation and to develop a product is to consider the existing links between Man and the product or more exactly Men and the product. What kind of links exist and are they voluntary links or not? In other words: has the "user" chosen to use the product that is in interaction with him or not? This question avoids having to identify all the users and split them between direct and indirect users.

\subsection{Indirect users}

In current everyday life every person has involuntary contact with products that they have not chosen despite the fact that this relation could be pleasant or uncomfortable. Many current examples can be found in transport design. In the street pedestrians are indirectly affected by the cars that they can see and hear and interact with when they cross the street. Furthermore, unfortunately, sometimes they could also be in more direct contact and be hurt by one of them.

Some pedestrians could also enjoy looking at a beautiful car, but the indirect user could also be indifferent to the product and not pay attention to it.

Actually we come across many products in our daily lives in this category and in order to be convinced of this it is sufficient to make a very simple experience: consider during only one day how many products you come into contact with of which you are an indirect user and compare to the number of products you use directly. Furthermore consider what kinds of attitude or feelings you have when you come into contact with these products: indifference, interest, happiness, horror, etc...

Regarding behavior and consequences, it is important to identify indirect users and their behavior and anticipate their roles and attitude to the product in the project management phase.

Indirect users could be interested by the product and appreciate looking at it or knowing more about it. Alternatively they could dislike or hate it and try to avoid contact or even oppose or fight against it's existence.

\subsection{Direct users}

Direct users are people who have voluntary chosen to be linked with the product. They are the persons whose requirements are generally taken into account by designers and ergonomists, but these are not the only kind of direct users so we have to enlarge the category to include other classes of direct users. At this first step we should observe that ergonomists and designers don't necessarily deal with the same types of direct users. Ergonomics, in general, looks at requirements of the person working the machine or device and product ergonomics deals with the worker or equivalent in the product specific case: the persons who are using the product to execute a task and reach a goal (P. Rabardel 1998). The Designer is trying to attract consumers and generally he adds to the functional approach that refers to the efficiency of the product, an emotional quality. Marketing, and more generally enterprises are looking for clients who are not necessary the future users. Many products are bought by one person, but are in fact going to be used by others.

We propose to define the direct users as people who have chosen to be linked to the product by a voluntary act: to buy, to choose, to use, which signifies that they have at one time or another a responsibility in the adoption of the decision to use the product. In this paper we will give a broad classification of direct users looking at the duties of designers and ergonomists.

\section{The importance of the direct + indirect user system}

We will end our explanation of the concept with an idea of what complex issues are linked to this dynamic system that refers amongst other things to ethics, law, economic success, brand value and quality management.

One first complex issue is the different role that the same person could have when confronted by the same kind of product. In a shop when the consumer looks for a product he is a possible client. If he buys it he becomes a client and if he uses it he also becomes a user. When he is still only looking at the product he is an indirect user and becomes a user when he decides to buy it. In all these steps he remains the same person but with a very different position and behavior. In transportation you are the direct user of your own car but the indirect user of the other cars around you.

Sometimes people give more importance to a product that they will never use than to those that they use themselves. Luxury products have more indirect users than direct users and this is still a reference, despite the fact that it could be a positive or negative reference. This product of reference leads 
the consumer to choose the same kind of product in terms of its aesthetics, function, and status. In a more detailed approach the usage value of the product could be appreciated by the direct user, but he could renounce using it because the prestige value given by indirect users to the product and its owner is low and gives the owner a bad image. Ergonomists know that workers don't like wearing some safety equipment such as gloves, shoes, safety hats and ear defenders because they think they look ridiculous wearing them. Depending on the local culture (C. Mourte 2008) most people like to follow the habits of the majority and adopt common standards meaning they give a strong importance to the indirect users point of view. Function, test, judgment, are linked to culture, and reciprocally products have influence on the culture of direct users but also on that of to indirect users as well.

The fashion success story consists of a wave of popularity that pushes indirect users to be attracted by the products adopted by the leaders or fashion icons, so that they then become direct users of these products by purchasing them.

Some environmental designs such as buildings, city infrastructure and equipment have more indirect users than direct users. The large numbers of direct and indirect users have different points of view and attribute different functions to the same product. An urban monument would have a symbolic function to one user and a very practical orientation function for a taxi driver.

Many examples can be found of product design projects that were defined taking into account the wishes of indirect users, such as the construction of cars, planes and trains which produce less noise and pollution of the environment, for example. These features, which may be considered an advantage for some indirect users, may however sometimes result in increased danger to others, since an elderly pedestrian may be unable to hear a quiet electric vehicle approaching in towns, with a consequent risk of being run over when he crosses the street.

Information systems designed for public transport using illuminated signs and loudspeaker messaging can also give complete guidance to the average direct user or passenger to find their next train or guide them to the appropriate exit, but can prove to be inadequate and confusing for those who have problems with their sight or hearing. Installing automatic glass doors and safety barriers on the open platforms of older metro lines is a positive safety feature, but can also result in additional sound reverberation in these areas making it more difficult for the visually im- paired to understand messages and detect corridor openings and exits (Uzan et al. 2011).

In a similar way wheelchair users are directly affected by the image created by their appearance and means of locomotion and the view of passers-by, whether they are expressed or not, has a direct effect on their self esteem. These passers-by are indirect users of the wheelchairs and their reactions are as important to the direct users of these chairs as the reaction of their peer group for teenagers wearing the latest fashion. It is not uncommon for the owner of a manual wheelchair to talk about how easy it is to maneuver his titanium-framed chair, or the owner of a power wheelchair to talk about how fast it can go and how quickly it can turn.

\section{Ergonomics and design responsibility and duty}

In both cases Ergonomists and Designers have professional objectives and a duty to look after users well being. For IEA “An ergonomist is an individual whose knowledge and skills concern the analysis of human-system interaction and the design of the system in order to optimize human well-being and overall system performance". For ICSID "Design is a creative activity whose aim is to establish the multifaceted qualities of objects, processes, services and their systems in whole life cycles. Therefore, Design is the central factor of innovative humanization of technologies".

To this kind of professional duty we could add the opening of perspectives by encouraging ideas, purpose and actions looking for equitable trade, sustainable development, international standards, total quality management and in particular we must mention international evolution.

The Federation for Alternative Trade, or International Fair Trade Association has established ten rules that define acceptable and unacceptable trade exchanges that have consequences for the designer's role and duties. The more evident one regarding both Ergonomics and Design is the working conditions: the salaries, health and safety of the workers involved in the product manufacturing and transportation; support for sexual equality and avoiding using children to do the work. Regarding this rule, workers must be considered as direct users that enlarge the field of direct users for the designer and push again the ergonomics of design for manufacturing defined by Martin Helander a long time ago. 


\section{Methodological approaches to find direct and indirect users}

The goal is to identify direct and indirect users, identify their needs and opinions; analyze contradictions and opposition and look for possible win/win situations. The problem is that no tools and methods have been specifically developed in order to identify direct and indirect users. Here we will propose extending some current tools to attain this goal.

6.1. Product life cycle analysis

It is the first stage in defining direct and indirect users and at the same time this approach is a central part of the philosophy of sustainable development. We have to consider all the stages in the lifecycle of the product that runs from the initial need to the disposal of the product. The question is: what are the positive and negative effects of each stage and for what kind of people and when?

Some examples of these stages:

- Products in action

What kind of people don't use the product and why? Is it because they don't want it or because they cannot use it? What kind of negative characteristics are associated with the use of the product; noise, smell, disturbing light effects, pollution. What kinds of person are affected by these problems now and in the future?

- Sales stage: the seller is a direct user and he needs to be confident of the product. People who accompany the buyer are indirect users. Many shopping centers have understood this and have installed playgrounds to keep their children occupied during the purchase. Children are indirect users of the product but direct users of the shopping center.

\subsection{Functional analysis}

To the classical question what is the product? What does it do? We could add: Who uses the product? What does it act on?

6.3. The Ergonomic approach and task analysis

The approach starts from the study of the activity in order to define a model of physical activity and the sensory and cognitive tasks required to achieve the user's desired result. It is necessary to identify unnecessary or excessive demands or features that are missing which could help the user.

6.4. Focus groups

This classical marketing tool could help to define the direct and indirect users of a specific product if this task is specifically included in the items to be discussed in the brain storming session. The chairman of the focus group needs to have guidelines to ensure that this question is not neglected.

\subsection{Standards and Regulations}

They exist to ensure respect for and protection of third parties, which include indirect users, but standards often follow events more than they lead. Standards are general in nature and insufficient and must be adapted case by case

6.6. Formalize the brief or specifications.

This is the first step in the job of the designer and is the meeting point of the requirements of every stakeholder. Direct and indirect users have to be mentioned in the brief followed by their needs, sentiments. This will help the project manager to define the product goals and constraints. The brief needs to offer a very realistic image of how the product functions in practice and give a scenario as an example of a typical user situation with criteria in order to asses if the direct and indirect needs are being met.

\subsection{Persona}

Designers like to work with this user representation. The problem is that at the moment this representation is only for one typical user. As in the cases previously cited of difficulties experienced by direct and indirect users suffering from sensory deficiencies it would be a considerable improvement to associate a main user or persona with the description of other persona or indirect users if not included already in the representation.

\section{Conclusions}

Design and Ergonomics work for the wellbeing of users. Quality management requires that all the stakeholders should be considered in the design process and that care should be taken to avoid providing an inadequate product or service for certain kinds of direct and indirect users. We need to develop new tools in order to find all the stakeholders for each product that is new or unusual and this is not easy to do. What are the features that can provide service and pleasure for all types of person susceptible to use or come into contact with a product, whatever their age, background, culture or limitations? Sustainable development demands a social consensus in the availability, adaptability, image and ease of use of all future products in a world evolving rapidly, both demographically and environmentally.

\section{References}

[1] Caliste J-P, Dejean P-H. Prise en Compte des Besoins des Utilisateurs, quels besoins, quels utilisateurs. Les Tech- 
niques de l'Ingénieur Paris 2012 Helander, M.G.(1995). A Guide to the Ergonomics of Manufacturing. London, UK: Taylor and Francis.

[2] Rabardel P; (1998) Des hommes et des Technologies. PUF Paris Fr
[3] Uzan, G. \& Wagstaff, P.R. A Model and Methods to solve problems of Accessibility and Information for the Visually Impaired. STHESCA, Conference, Krakow July 2011. 